\title{
Surface proximity and boron concentration effects on end-of-range defect formation during nonmelt laser annealing
}

\author{
J. A. Sharp, ${ }^{1, a)}$ A. J. Smith, ${ }^{1}$ R. P. Webb, ${ }^{1}$ K. J. Kirkby, ${ }^{1}$ N. E. B. Cowern, ${ }^{2}$ D. Giubertoni, ${ }^{3}$ \\ S. Gennaro, ${ }^{3}$ M. Bersani, ${ }^{3}$ M. A. Foad, ${ }^{4}$ P. F. Fazzini, ${ }^{5}$ and F. Cristiano ${ }^{6}$ \\ ${ }^{1}$ Surrey Ion Beam Centre, Advanced Technology Institute, Faculty of Engineering and Physical Sciences, \\ University of Surrey, Guildford GU2 7XH, United Kingdom \\ ${ }^{2}$ School of Electrical, Electronic and Computer Engineering, University of Newcastle upon Tyne, \\ Newcastle upon Tyne NE1 7RU, United Kingdom \\ ${ }^{3}$ Fondazione Bruno Kessler, Ricerca Scientifica e Tecnologica, Via Sommarive 18, Povo (Trento) 38050, \\ Italy \\ ${ }^{4}$ Front End Products, Applied Materials Inc., 974 E. Arques Avenue, Sunnyvale, California 94086, USA \\ ${ }^{5}$ CEMES-CNRS, University of Toulouse, 29, rue Jeanne Marvig, 31055 Toulouse, France \\ ${ }^{6}$ LAAS-CNRS, University of Toulouse, 7 av. Du Col. Roche, 31077 Toulouse, France
}

(Received 28 November 2007; accepted 2 February 2008; published online 28 February 2008)

\begin{abstract}
The effects of surface proximity and B concentration on end-of-range defect formation during nonmelt laser annealing in preamorphized silicon have been studied. These effects were analyzed by observing the activation and diffusion of an ultrashallow B implant, using Hall effect and secondary ion mass spectrometry measurements. By adjusting the preamorphizing implant and laser annealing conditions, B deactivation and diffusion were minimized, resulting in a sheet resistance of $\sim 600 \Omega$ /sq with a $16 \mathrm{~nm}$ junction depth. This is attributed to a combination of enhanced dissolution of end-of-range defects and preferential formation of B-interstitial clusters due to the surface proximity and high B concentration, respectively. () 2008 American Institute of Physics.

[DOI: $10.1063 / 1.2885091]$
\end{abstract}

As complementary metal-oxide-semiconductor devices are continually downscaled, their source/drain extension regions require ultrashallow junction depths with high electrical activation. A common technique to produce highly electrically active $p$-type junctions is to preamorphize the surface region with a relatively high mass ion, typically Ge, and then implant low energy B into the amorphous region. During subsequent annealing, solid phase epitaxial regrowth (SPER), transforms this region back to its original crystalline structure, with the B incorporated substitutionally at concentrations well above its equilibrium solid solubility limit. ${ }^{1}$ However, excess Si interstitials remaining just beyond the original amorphous/crystalline $(a / c)$ interface position condense into clusters which then evolve by Ostwald ripening ${ }^{2}$ into extended defects known as the end-of-range (EOR) defect band. During annealing, $\mathrm{Si}$ interstitials are released from the EOR defect band. Their coupling with B leads to transient enhanced diffusion (TED) and boron-interstitial cluster (BIC) formation, leading to increased junction depth and reduced dopant electrical activation, ${ }^{3,4}$ respectively. One promising way to overcome these problems is to reduce the coupling between interstitials and B atoms by annealing for a short time at high temperature. ${ }^{5}$ The ultimate way in which to do this is to anneal with a submelt laser. ${ }^{6}$ This approach has been proven in practice to reduce EOR defect density and TED. ${ }^{\dagger}$ In this letter, submelt laser annealing is employed in a different approach where EOR defect behavior during laser and further annealing is investigated when the position of the $a / c$ interface is adjusted with respect to a $\mathrm{B}$ implant.

Three $n$-type (100) Czochralski-silicon wafers were preamorphized with 2 or $5 \mathrm{keV} \mathrm{Ge}^{+}$to a dose of 5 $\times 10^{14} \mathrm{~cm}^{-2}$, or $10 \mathrm{keV} \mathrm{Ge}$, to a dose of $1 \times 10^{15} \mathrm{~cm}^{-2}$ pro-

${ }^{a)}$ Electronic mail: j.sharp@surrey.ac.uk. ducing surface amorphous layer thicknesses of 7, 14, and $22 \mathrm{~nm}$, respectively, (measured by Rutherford backscattering spectroscopy ${ }^{8}$ ). B was subsequently implanted into these amorphous layers at a fixed energy and dose of $500 \mathrm{eV}$ and $2 \times 10^{15} \mathrm{~cm}^{-2}$, respectively, with $0^{\circ}$ tilt and twist. A millisecond annealing was performed on the wafers, using a scanning diode laser with the surface reaching a peak temperature of $1150{ }^{\circ} \mathrm{C}$. Three strips were annealed across each wafer; each receiving either one, five or ten laser scans. All amorphous layers regrew by SPER during the initial laser scan. The kinetic Monte Carlo implant simulator $\mathrm{KING}^{9}$ was used to identify Ge energies and doses that would position the $a / c$ interface in three alternative depths: well beyond the expected depth of the B implant profile, close to the profile, and within the peak of the boron profile. These successively shallower depths correspond to an increase in B concentration in the EOR region from below $10^{18}$ to $\sim 6 \times 10^{20} \mathrm{~cm}^{-3}$ as subsequently measured by secondary ion mass spectroscopy (SIMS). The simulated net excess of $\mathrm{Si}$ interstitials beyond the $a / c$ interface was found to be independent of the implant condition within $\pm 5 \%$. Van der Pauw and Hall Effect measurements were used to measure the sheet resistance $\left(R_{s}\right)$ and the electrically active B fraction and mobility were extracted assuming a Hall scattering factor of unity.

The effect of the laser annealing step is examined initially. Figure 1(a) shows the electrically active dose of B as a function of Ge energy (EOR position) and number of laser scans. Comparing the values for the three Ge PreAmorphizing Implant (PAI) energies, one can see significant difference in electrical activation after one laser scan. At $10 \mathrm{keV}$, where the EOR damage would be furthest away from the surface and in the region of lowest B concentration, the B implant has the highest initial active dose of $\sim 4.9$ $\times 10^{14} \mathrm{~cm}^{-2}$. A similar value, $4.6 \times 10^{14} \mathrm{~cm}^{-2}$, is obtained 


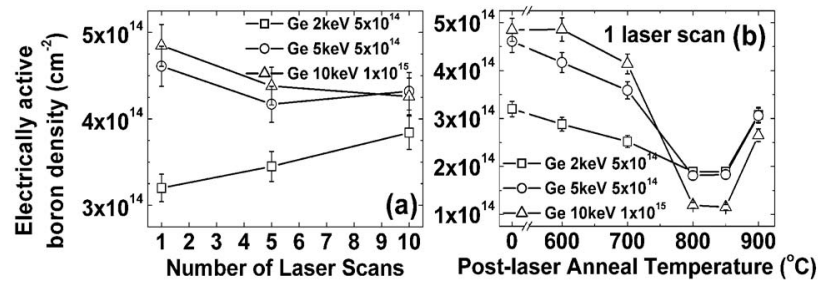

FIG. 1. Electrically active boron density of a $500 \mathrm{eV}, 2 \times 10^{15} \mathrm{~cm}^{-2}$ boron implant into preamorphized silicon (a) as a function of laser scans and Ge PAI energy and (b) for the 2, 5, and $10 \mathrm{keV}$ Ge PAI conditions after laser annealing with one scan and postlaser annealing.

when the Ge PAI energy is reduced to $5 \mathrm{keV}$, but in the $2 \mathrm{keV}$ PAI case the electrical activation is $\sim 30 \%$ lower, at $3.2 \times 10^{14} \mathrm{~cm}^{-2}$. As the number of laser scans is increased to five and then ten scans, it can be seen that the electrical activation in each Ge PAI sample trends toward the same value.

As the situation after one laser scan shows a substantial variation compared to five and ten laser scans, this one-scan case is focused on. Figure 2(a) shows B diffusion for this case, measured by SIMS. A Cameca Wf SC-ULTRA tool was used, running a $500 \mathrm{eV} \mathrm{O}+$ primary beam at a $68^{\circ}$ angle of incidence, with oxygen leak. Stage rotation was used to prevent ripple formation. ${ }^{10}$ Corresponding sheet resistance values for each PAI condition are included for reference. Since the as-implanted B profiles for the different Ge PAI conditions show negligible differences in terms of depth and distribution, only one as-implanted profile is shown. The $2 \mathrm{keV}$ Ge PAI sample shows virtually no diffusion after one laser scan combined with the highest $R_{s}$, however, the 5 and $10 \mathrm{keV}$ Ge PAI samples show a more appreciable diffusion and much lower $R_{s}$. The lowest $R_{s}$ is found in the $10 \mathrm{keV}$ sample, together with evidence of "concentration-enhanced diffusion" indicating a higher level of substitutional B in the deeper part of the $\mathrm{B}$ profile peak. In the $5 \mathrm{keV}$ Ge PAI sample, where the tail of the B profile overlaps the EOR region, it is possible to see, indirectly, a degree of B trapping at a depth of $\sim 16 \mathrm{~nm}$. ${ }^{11}$ The respective Hall mobilities are $21.9,19.9$, and $21.4 \mathrm{~cm}^{2} / \mathrm{V} \mathrm{s}$ for the 10,5 , and $2 \mathrm{keV} \mathrm{Ge}$ PAI samples, respectively, which show that the trend in $R_{s}$ is driven by changes in B electrical activation.

Turning now to the situation after ten laser scans, where the electrical properties for the different PAI conditions have converged to almost the same values, it can be seen that the diffusion in the $2 \mathrm{keV}$ sample is much less than in the 5 or $10 \mathrm{keV}$ samples, resulting in a shallower junction depth [Fig. 2(b)].

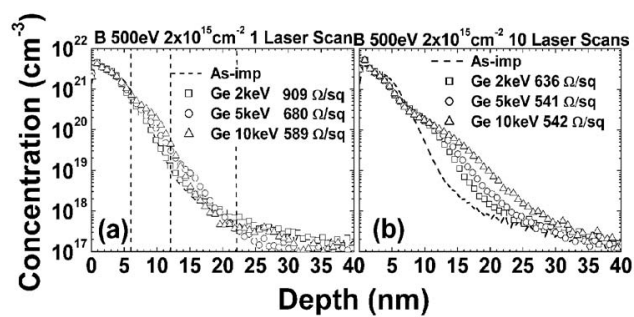

FIG. 2. Boron SIMS profiles and sheet resistances after laser annealing with (a) one scan and (b) ten scans for the three Ge PAI conditions, showing the corresponding positions of the $a / c$ interface after implantation (dashed ver-

tical lines).
Downloaded 30 Mar 2009 to 131.227.178.132. Redistribution subject to AlP license or copyright; see http://apl.aip.org/apl/copyright.jsp

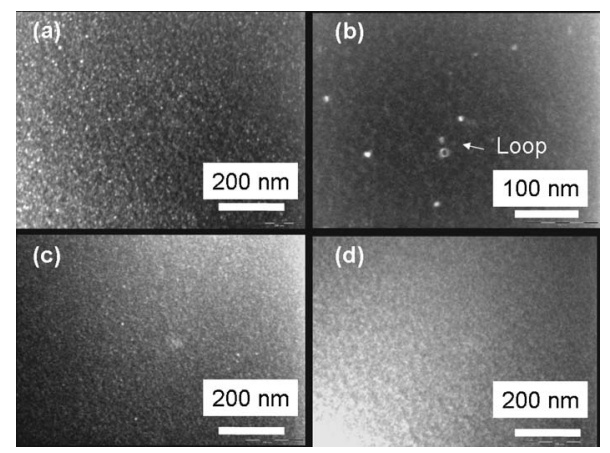

FIG. 3. Plan-view TEM images in Weak Beam Dark Field condition with $g=[422]$ and $s$ slightly positive. (a) and (b) correspond to sample preamorphized with Ge at $10 \mathrm{keV}$ and laser annealed with one and ten scans. (c) and (d) corresponds to $\mathrm{Ge}$ at $2 \mathrm{keV}$, one and ten scans.

The thermal stability of activated junctions can indirectly reveal the presence of EOR defects through a large amount of $\mathrm{B}$ deactivation during postactivation annealing. 4 To investigate this, a $60 \mathrm{~s}$ isochronal annealing schedule from 600 to $900{ }^{\circ} \mathrm{C}$ with a $50{ }^{\circ} \mathrm{C} / \mathrm{s}$ heating ramp rate was carried out to examine the deactivation of the $\mathrm{B}$ after one laser scan and is shown in Fig. 1(b). When the postlaser rapid thermal annealing temperature is increased to $800{ }^{\circ} \mathrm{C}$ the electrically active dose drops significantly, consistent with BIC formation. ${ }^{4}$ This deactivation is greatest in the $10 \mathrm{keV}$ sample, but the consistently lower electrical activation in the $2 \mathrm{keV}$ sample at up to $700{ }^{\circ} \mathrm{C}$ shows that a greater fraction of $\mathrm{B}$ is clustered than in the other two conditions.

In order to clarify the defect density after laser annealing, transmission electron microscopy (TEM) was used to examine the EOR defects; the results are illustrated in Fig. 3. Images were taken in weak beam dark field conditions using $\mathrm{B}=[113]$ with $g=\langle 422\rangle$ to increase defect contrast (visibility improvement) and the number of visible defect variants (increased apparent density). ${ }^{12}$ After laser annealing with one scan, large densities of silicon interstitial clusters are observed in the 5 and $10 \mathrm{keV}$ [Fig. 3(a)] samples, which represents a potential source of interstitials which accounts for the considerable deactivation during subsequent annealing. However, for the $2 \mathrm{keV}$ sample, where the $\mathrm{B}$ activation and diffusion were minimal, Fig. 3(c) shows that very few TEMvisible defects have formed. This reduction in EOR formation is thought to be due to a combination of two effects.

(i) The concentration gradient of Si interstitials between the EOR defect band and the surface will be higher due to the proximity of the surface. ${ }^{3}$

(ii) As the excess interstitials overlap strongly with the as-implanted B profile, the EOR defects that are formed may consist of BICs rather than predominantly self-interstitial defects.

Following the line of thought in (ii), it is worth noting that BICs are much more stable than self-interstitial defects of comparable size and, as a result, they act as a temporary sink for interstitials, while at the same time there is less driving force for them to ripen into TEM-visible defects. This interpretation agrees with an earlier study by Haynes et al. ${ }^{13}$ who showed that the density of (TEM-visible) EOR defects reduced during annealing in the presence of $\mathrm{B}$ and that BICs 
were preferentially formed. Another consequence of the sinking of interstitials during BIC formation would be a reduction in boron diffusion, consistent with the data in Fig. 2. After laser annealing with ten scans, there is a drastic decrease in the number of defects in the 5 and $10 \mathrm{keV}$ samples and in the $10 \mathrm{keV}$ case [Fig. 3(b)] some dislocation loops have formed. In contrast, there are no visible defects in the $2 \mathrm{keV}$ sample after ten laser scans [Fig. 3(d)].

To summarize, the effect of the position of the $a / c$ interface with respect to the surface and boron concentration on EOR defect formation has been studied after nonmelt laser and prolonged annealing. A combination of mechanisms appears to be acting to eliminate TEM-visible EOR defects and prevent significant diffusion and deactivation of the ultrashallow B implant. When the $a / c$ interface and, hence, EOR defect band are positioned close to the silicon surface within a high concentration of boron, EOR defect formation during a laser annealing of one scan at $1150{ }^{\circ} \mathrm{C}$ is modulated by the increased proximity of the surface which can be considered as an interstitial sink and by the preferential formation of BICs rather than predominantly selfinterstitial EOR defects. Furthermore, after ten scans the $2 \mathrm{keV}$ condition has the lowest defect density, least amount of diffusion, and the location of the BIC-rich EOR band is within the peak of the B profile, thus, avoiding potential issues with junction leakage while not having any significant effect on mobility. This promises to be a viable method of forming stable, highly active, ultrashallow junctions.

The authors would like to acknowledge the U.K. Engineering and Physical Sciences Research Council and the Sur- rey Ion Beam Centre for their support. F. Cristiano, P. F. Fazzini, and N. E. B. Cowern wish to acknowledge the support of the European integrated project PullNANO.

${ }^{1}$ J.-Y. Jin, J. Liu, U. Jeong, S. Mehta, and K. Jones, J. Vac. Sci. Technol. B 20, 422 (2002).

${ }^{2}$ C. Bonafos, D. Mathiot, and A. Claverie, J. Appl. Phys. 83, 3008 (1998).

${ }^{3}$ N. E. B. Cowern, D. Alquier, M. Omri, A. Claverie, and A. Nejim, Nucl. Instrum. Methods Phys. Res. B 148, 257 (1999).

${ }^{4}$ B. J. Pawlak, R. Surdeanu, B. Colombeau, A. J. Smith, N. E. B. Cowern, R. Lindsay, W. Vandervorst, B. Brijs, O. Richard, and F. Cristiano, Appl. Phys. Lett. 84, 2055 (2004).

${ }^{5}$ A. E. Michel, W. Rausch., P. A. Ronsheim, and R. H. Kasti, Appl. Phys. Lett. 50, 416 (1987).

${ }^{6}$ S. Earles, M. Law, K. Jones, S. Talwar and S. Corcoran, Si Front-End Processing-Physics and Technology of Dopant-Defect Interactions III, MRS Symposia Proceedings No. 669 (Materials Research Society, Pittsburgh, 2001), p. J4.1.1.

${ }^{7}$ J. A. Sharp, N. E. B. Cowern, R. P. Webb, K. J. Kirkby, D. Giubertoni, S. Gennaro, M. Bersani, M. A. Foad, F. Cristiano, and P. F. Fazzini, Appl. Phys. Lett. 89, 192105 (2006).

${ }^{8}$ J. J. Hamilton, E. J. H. Collart, B. Colombeau, C. Jeynes, M. Bersani, D. Giubertoni, J. A. Sharp, N. E. B. Cowern, and K. J. Kirkby, Nucl. Instrum. Methods Phys. Res. B 237, 107 (2005).

${ }^{9}$ G. Lulli, M. Biaconi, R. Nipoti, E. Albertazzi, M. Cervera, A. Carnera, and C. Cellini, J. Appl. Phys. 82, 5958 (1997).

${ }^{10}$ M. Bersani, D. Giubertoni, E. Iacob, M. Barozzi, S. Pederzoli, L. Vanzetti, and M. Anderle, Appl. Surf. Sci. 252, 7315 (2006).

${ }^{11}$ B. Colombeau, A. J. Smith, N. E. B. Cowern, B. J. Pawlak, F. Cristiano, R. Duffy, A. Claverie, C. J. Ortiz, P. Pichler, E. Lampin, and C. Zechner, Silicon Front-End Junction Formation-Physics and Technology, MRS Symposia Proceedings No. 810 (Materials Research Society, Pittsburgh, 2004), p. 91.

${ }^{12}$ S. Boninelli, N. Cherkashin, A. Claverie, and F. Cristiano, Appl. Phys. Lett. 89, 161904 (2006).

${ }^{13}$ T. E. Haynes, D. J. Eaglesham, P. A. Stolk, H.-J. Gossmann, D. C. Jacobson, and J. M. Poate, Appl. Phys. Lett. 69, 1376 (1996). 